\title{
OBESIDADE EM MULHERES PÓS-CIRURGIA DE CÂNCER DE MAMA PARTICIPANTES DE HIDROTERAPIA
}

Mariana Romanholi Palma ${ }^{1}$, Fernanda Elisa Ribeiro ${ }^{2}$, Elisa Bizetti Pelai ${ }^{1}$, Alessandra Madia Mantovani Fabri ${ }^{3}$, Mariane Fátima da Silva Araujo ${ }^{3}$, Lara Nery Peixoto ${ }^{3}$, Carolina Andrade Egydio ${ }^{3}$, Edna Maria do Carmo ${ }^{4}$, Cristina Elena Prado Teles Fregonesi ${ }^{4}$

Universidade Estadual Paulista - FCT/UNESP. ${ }^{1}$ Programa de Pós-Graduação Stricto Sensu (nível mestrado) em Fisioterapia. ${ }^{2}$ Programa de Pós-Graduação Lato Sensu em Fisioterapia. ${ }^{4}$ Departamento de Fisioterapia, Presidente Prudente, SP. ${ }^{3}$ Universidade Estadual Paulista - UNESP. Programa de Pós-Graduação Stricto Sensu (nível doutorado) do Instituto de Biociências, Rio Claro, SP. e-mail: marianaromanholi@hotmail.com

\section{RESUMO}

Obesidade e sobrepeso são fatores de risco para desenvolvimento de neoplasias e oferecem maiores chances de recorrência e progressão. O objetivo deste estudo foi verificar a presença desses fatores de risco em mulheres pós-cirurgia de câncer de mama, participantes de hidroterapia, por meio do índice de massa corporal (IMC) e circunferência da cintura. Foram coletados massa corporal e estatura para cálculo do IMC e circunferência da cintura. Participaram

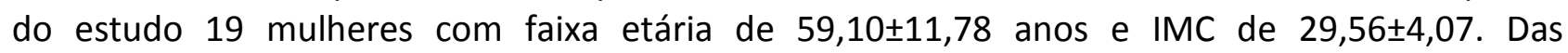
participantes, $94,74 \%$ apresentaram-se obesas ou com sobrepeso e 78,94\% apresentaram circunferência da cintura maior ou igual a 88 centímetros. São necessárias readaptações do tratamento e orientações sobre malefícios da obesidade nesta população. As precauções devem estar dentre os objetivos de saúde pública, não apenas como forma de evitar recidivas, mas também como prevenção de patologias que a tem como fator de risco.

Palavras-chave: Obesidade, Sobrepeso, Câncer de mama, Circunferência da cintura, Índice de Massa Corporal

\section{OBESITY IN WOMEN AFTER SURGERY FOR BREAST CANCER PARTICIPANTS OF HYDROTHERAPY}

\begin{abstract}
Obesity and overweight is a risk factor for developing cancer and offers a greater chance of recurrence and progression. The aim of this study was to verify the presence of these risk factors in women post surgery for breast cancer, participants hydrotherapy through the body mass index (BMI) and waist circumference. Were collected body mass and height to calculate $\mathrm{BMI}$ and waist circumference. The study included 19 women with age of $59.10 \pm 11.78$ years and BMI 29.56 \pm 4.07 . Of the participants, $94.74 \%$ presented obesity or overweight and $78.94 \%$ presented waist circumference greater than or equal to 88 centimetre. Adaptations of treatment and guidance about harm of obesity in this population are needed. Precautions should be among the goals of public health not only as a way to prevent relapses, but also as prevention of diseases that have obesity as a risk factor.
\end{abstract}

Key- words: Obesity, Overweight, Breast Câncer, Waist Circumference, Body Mass Index 


\section{INTRODUÇÃO}

A obesidade é acúmulo anormal ou excessivo de tecido adiposo ${ }^{(1,2)}$, classificada por índice de massa corporal (IMC) igual ou superior a $30 \mathrm{~kg} / \mathrm{m}^{2(3)}$. Estar obeso ou com sobrepeso contribui para o aumento do risco de desenvolvimento de diversas doenças, dentre elas, as neoplasias ${ }^{(4-6)}$.

O câncer de mama é o segundo mais incidente entre todas as neoplasias no mundo e o mais frequente entre as mulheres $^{(7)}$, tem elevada mortalidade e representa um importante problema de saúde pública ${ }^{(8)}$.

A adiposidade abdominal, indicada pela circunferência da cintura, é uma medida adicional que verifica a distribuição da gordura corporal $^{(3)}$. Em níveis elevados, está associada a maior risco de câncer de mama em mulheres pós-menopausa ${ }^{(9)}$, o que pode ser explicado devido ao tecido adiposo ser a principal fonte de síntese de estrogênio nesta fase ${ }^{(1)}$. Estudos sugerem que o excesso de peso oferece maiores chances de recorrência, progressão e pior prognóstico do câncer ${ }^{(4,10)}$.

Conhecendo os possíveis malefícios da obesidade e do sobrepeso, sabendo que tratam-se de fatores de risco modificáveis, o objetivo deste estudo foi verificar a presença desses fatores em mulheres pós cirurgia de câncer de mama participantes de hidroterapia, por meio do índice de massa corporal (IMC) e circunferência da cintura.

\section{METODOLOGIA}

Trata-se de um estudo transversal, desenvolvido no Centro de Estudos e Atendimento em Fisioterapia e Reabilitação (CEAFIR) da Faculdade de Ciências e Tecnologia (FCT) - Universidade Estadual Paulista (UNESP), Campus de Presidente Prudente. Foi aprovado pelo Comitê de Ética em Pesquisa da FCT/UNESP (CAAE: 32108414.8.0000.5402). As participantes receberam esclarecimentos sobre os procedimentos e objetivos da pesquisa e, após concordarem em participar, assinaram o Termo de Consentimento Livre e Esclarecido (TCLE). Participaram do estudo mulheres pós tratamento cirúrgico de câncer de mama, frequentadoras de aulas de hidroterapia duas vezes semanais, por no mínimo 6 meses.

Foram coletados dados como massa corporal e estatura, para cálculo do IMC [peso $(\mathrm{kg}) /$ altura $^{2}\left(\mathrm{~m}^{2}\right)$ ], e circunferência da cintura. A coleta foi realizada em balança digital com estadiômetro (Welmy ${ }^{\circledR} \mathrm{W} 110 \mathrm{H}$, Brasil). O IMC foi classificado de acordo com a Organização Mundial da Saúde ${ }^{(3)}$ em: $<18,5$ $\mathrm{kg} / \mathrm{m}^{2}$ baixo peso; entre 18,5 a $24,9 \mathrm{~kg} / \mathrm{m}^{2}$ peso ideal; 25,0 a $29,9 \mathrm{~kg} / \mathrm{m}^{2}$ excesso de peso; $\geq 30,0 \mathrm{~kg} / \mathrm{m}^{2}$ obesidade.

A circunferência da cintura foi verificada em centímetros $(\mathrm{cm})$, por meio de 
uma fita métrica flexível e inextensível, com precisão de $01 \mathrm{~mm}$, entre o ponto médio da distância da crista ilíaca e rebordo costal inferior $^{(11)}$. Os valores menores que $80 \mathrm{~cm}$ são considerados normais, entre $80-87 \mathrm{~cm}$ como risco aumentado de complicações associadas à obesidade e valores maiores que $88 \mathrm{~cm}$ como risco muito aumentado ${ }^{(11-13)}$.

Os dados foram apresentados de maneira descritiva, por média, desvio padrão e porcentagem.

\section{RESULTADOS}

Participaram do estudo 19 mulheres submetidas à procedimento cirúrgico para retirada de câncer de mama, participantes de hidroterapia. A faixa etária média foi de $59,10 \pm 11,78$ anos e IMC médio foi de 29,56 $\pm 4,07$.

Tabela 1. Índice de massa corporal (IMC) de mulheres pós cirurgia de câncer de mama, participantes de hidroterapia, apresentado em porcentagem (\%). n=19.

\begin{tabular}{cc}
\hline IMC & $\%$ \\
\hline Baixo peso & 0 \\
Peso Ideal & 5,26 \\
Sobrepeso & 52,63 \\
Obesidade & 42,11
\end{tabular}

Quanto à circunferência da cintura, $78,94 \%$ das mulheres apresentaram medidas maiores ou iguais a $88 \mathrm{~cm}, 10,53 \%$ entre 80 $87 \mathrm{~cm}$ e $10,53 \%$ menores que $80 \mathrm{~cm}$.

\section{DISCUSSÃO}

O impacto da obesidade sobre o risco e progressão do câncer de mama pode depender dos níveis de adiposidade que a mulher está exposta ${ }^{(15)}$. O IMC elevado está associado a piores prognósticos, a longo prazo, em mulheres diagnosticadas com a doença, que pode ser explicado devido ao excesso de peso levar a um crescimento tumoral mais rápido ${ }^{(16)}$.

Das mulheres pós cirurgia de câncer de mama participantes deste estudo, $94,74 \%$ apresentaram-se acima do peso ou com obesidade e $78,94 \%$ apresentaram circunferência da cintura maior ou igual a 88 cm, demonstrando a concentração de adiposidade em região abdominal.

A adiposidade excessiva leva a um aumento da atividade dos hormônios sexuais, principalmente do estrogênio, aumentando o risco de câncer de mama ${ }^{(14,17)}$. Além disso, o aumento da concentração de 
insulina, que pode ocorrer em mulheres obesas, tem sido considerado como estimulador do crescimento de células neoplásicas em casos de câncer de mama ${ }^{(14,18)}$

É certo que a obesidade é um fator modificável e que merece atenção. 0 profissional de saúde tem como dever alertar e esclarecer sobre os malefícios da obesidade, assim como sugerir mudanças no estilo de vida, modificar as atividades aplicadas de acordo com a necessidade das pacientes e recomendar a redução de peso e acompanhamento nutricional como parte de um tratamento global ${ }^{(5)}$.

As precauções quanto à obesidade devem estar dentre os objetivos de saúde pública, não apenas como forma de evitar recidivas em pessoas que já tiveram a patologia, mas também como prevenção de alguns tipos de neoplasias que a tem como fator de risco.

\section{CONCLUSÃO}

A maioria das participantes do estudo apresentou sobrepeso ou obesidade, bem como adiposidade central, o que sugere que aulas de hidroterapia realizadas duas vezes por semana não tem sido suficiente para combater esse fator de risco, demonstrando a necessidade de readaptações do tratamento, inclusão de acompanhamento nutricional e orientações sobre malefícios da obesidade.

\section{REFERÊNCIAS}

1. Khan S, Shukla S, Sinha S, Meeran SM. Role of adipokines and cytokines in obesityassociated breast cancer: therapeutic targets. Cytokine Growth Factor Rev. 2013;24(6):50313.

http://dx.doi.org/10.1016/i.cytogfr.2013.10.0 $\underline{01}$

2. Liu LN, Miaskowski C, Wang JS, Chen SC, Chen ML. Accuracy of body mass index to determine obesity in women with breast cancer: An observational study of Taiwanese sample. International Journal of Nursing Studies. 2010; 47(8): 994-1000. http://dx.doi.org/10.1016/j.ijnurstu.2010.01. $\underline{002}$

3. World Health Organization (WHO). Waist circumference and waist-hip ratio: report of a WHO expert consultation. Geneva: World Health Organization. 2008:811.

4. Renehan AG, Tyson $M$, Egger $M$, Heller RF, Zwahlen M. Body-mass index and incidence of cancer: a systematic review and meta-analysis of prospective observational studies. The lancet. 2008; 371 (9612): 56978.

http://dx.doi.org/10.1016/S01406736(08)60269-X

5. Robinson PJ, Bell RJ, Davis SR. Obesity is associated with a poorer prognosis in women with hormone receptor positive breast câncer. Maturitas. 2014. http://dx.doi.org/10.1016/j.maturitas.2014.0 $\underline{7.004}$

6. Goodwin PJ, Stambolic V. Obesity and insulin resistance in breast cancer-chemoprevention strategies with a focus on metformin. Breast. 2011; 20 Suppl 3:S31-5. 
http://dx.doi.org/10.1016/S0960-

\section{6(11)70291-0}

7. International Agency for Research on Cancer.Latest world cancer statistics global cancer burden rises to 14.1 million new cases in 2012: Marked increase in breast cancers must be addressed. 2013; http://www.iarc.fr/en/mediacentre/pr/2013/pdfs/pr223 E.pdf

8. Oshiro $M L$, Bergmann $A$, Silva RG, Costa KC, Travaim IEB, Silva GB, et al. Câncer de Mama Avançado como Evento Sentinela para Avaliação do Programa de Detecção Precoce do Câncer de Mama no Centro-Oeste do Brasil. Revista Brasileira de Cancerologia. 2014; 60(1):15-23.

9. Cleary MP, Grossmann ME. Obesity and Breast Cancer: The Estrogen Connection. Endocrinology. 2009; 150(6): 2537-42. http://dx.doi.org/10.1210/en.2009-0070

10. Jain $R$, Strickler HD, Fine $E$, Sparano JA. Clinical studies examining the impact of obesity on breast cancer risk and prognosis. J Mammary Gland Biol Neoplasia. 2013;18(34):257-66. http://dx.doi.org/10.1007/s10911013-9307-3

11. Felden, JBB, Figueiredo, ACL. Ciência da saúde coletiva [online]. 2011;16(5): 242533.

12. Lean MEJ, Morrison CE, Han TS. Waist circumference as a measure for indicating need for weight management. BMJ 1995; 311:158-61.

http://dx.doi.org/10.1136/bmj.311.6998.158

13. Peixoto MRG, Benício MHD, Latorre MRDO, Jardim PCBV. Circunferência da cintura e índice de massa corporal como preditores da hipertensão arterial.Arquivos Brasileiros de Cardiologia.2006;87(4): 46270. http://dx.doi.org/10.1590/s0066$\underline{782 \times 2006001700011}$
14. Pinheiro RL, Sarian LO, Pinto-Neto AM, Morais S, Costa-Paiva L. Relationship between body mass index, waist circumference and waist to hip ratio and the steroid hormone receptor status in breast carcinoma of pre- and postmenopausal women. Breast. 2009;18(1):8-12. http://dx.doi.org/10.1016/i.breast.2008.09.0 $\underline{01}$

15. Perks CM, Holly JM. Hormonal mechanisms underlying the relationship between obesity and breast cancer. Endocrinol Metab Clin North Am. 2011;40(3):485-507.

http://dx.doi.org/10.1016/j.ecl.2011.05.010

16. Kamineni A, Anderson $\mathrm{ML}$, White E, Taplin SH, Porter P, Ballard-Barbash R, et al. Body mass index, tumor characteristics, and prognosis following diagnosis of earlystage breast cancer in a mammographically screened population. Cancer Causes Control. 2013;24(2):305-12.

http://dx.doi.org/10.1007/s10552-012-0115$\underline{7}$

17. Key TJ. Endogenous o estrogens and breast cancer risk in premenopausal and postmenopausal women. Steroids. 2011 Jul;76(8):812-5.

http://dx.doi.org/10.1016/i.steroids.2011.02. $\underline{029}$

18. Agnoli $C$, Berrino $F$, Abagnato CA, Muti $P$, Panico $S$, Crosignani $P$, et al. Metabolic syndrome and postmenopausal breast cancer in the ORDET cohort: a nested case-control study. Nutr Metab Cardiovasc Dis. 2010;20(1):41-8.

http://dx.doi.org/10.1016/j.numecd.2009.02. $\underline{006}$

Recebido para publicação em 19/08/2014

Revisado em 03/09/2014

Aceito em 06/09/2014 